\title{
AXILLARY LYMPH NODE DISSECTION IN BREAST CANCER PATIENTS. THE ROLE OF SENTINEL LYMPH NODE BIOPSY
}

\author{
Nicolae Bacalbasa ${ }^{1}$, Olivia Ionescu ${ }^{2}$, Irina Balescu ${ }^{3}$ \\ 1 "Carol Davila" University of Medicine and Pharmacy, Bucharest \\ 2"Bucur" Maternity Hospital, Bucharest \\ 3"Ponderas" Hospital, Bucharest
}

\begin{abstract}
Rationale. Since its introduction in the early 1990s, sentinel lymph node biopsy (SLNB) is regarded as the standard treatment for patients with clinically negative axillary lymph nodes (LNs) on initial presentation. Classically, when the SLN biopsy is negative, the axillary LN dissection (ALND) is no further necessary. On the other hand, performing complete ALND in case of a positive SLNB is controversial, recent data from randomized controlled studies suggesting that, in these cases, the tumor biology has a greater impact on the adjuvant treatment decision than the completion of an ALND.

Objective. The aim of this review is to ascertain whether axillary LN surgery has survival benefits in women with early breast cancer and SLN involvement, either micro-metastatic or macro-metastatic. Moreover, it tries to assess the value of SLN biopsy before and after primary systemic chemotherapy and its role in the staging of the axilla in locally recurrent breast cancer.

Materials and method. We searched Pubmed, Medline, the Cochrane Register of Controlled Trials and G.I.N (Guidelines International Network) databases for English language articles about the need of ALND in women with both positive and negative SLNs using controlled vocabulary (e.g. "breast cancer") and key words (e.g "sentinel lymph node", "axilla dissection"). The analysis was restricted to retrospective studies and randomized controlled trials focusing on survival benefits in terms overall (OS) or disease-free survival (DFS). Conclusions. There is increasing evidence which indicates that ALND can be avoided in a specific group of patients with early breast cancer, even though the SLNB is positive. A correlation between the clinico-pathological features of the breast cancer and the probability of residual disease in the axilla, could allow the selection of cases in which ALND can be omitted. In the context of neo-adjuvant chemotherapy, it is not yet established if positive SLNs could be converted to negative SLNs after chemotherapy as the rate of false-negative results is still high.
\end{abstract}

Keywords: sentinel lymph node, breast cancer, biopsy, axillary dissection

\section{Abbreviations:}

SLNB = sentinel lymph node biopsy; LN = lymph node; ALND = axillary lymph node dissection; $\mathrm{ALN}=$ axillary lymph node; OS = overall survival; DFS = disease-free survival; US = ultrasound

\section{INTRODUCTION - THE CONCEPT OF SENTINEL LYMPH NODE}

Over the past century, a less radical and mutilating surgical treatment of the breast cancer has been progressively embraced by the clinicians basing on the favorable outcomes in terms of morbidity rates and life quality obtained with the introduction of new treatment methods such as systemic chemotherapy, radiation or the SLNB concept. The model proposed by Fisher emphasizes that breast cancer is mainly a systemic disease from an early point in time so that less aggressive surgery should be recommended. The achievement of a lower residual tumor burden results in a lower risk of spontaneously activating resistant tumor clones. (1) 
The treatment protocol of the breast cancer has changed from the radical mastectomy by Halsted and modified mastectomy by Patey and Dyson to a more conservative surgical approach such lumpectomy, SLN biopsy and/or axilla dissection. (2)

This progress has been possible due to an increasing awareness of the female population of the necessity of breast cancer screening which has resulted in an earlier detection, and possibility of treatment with novel targeted chemotherapeutic agents. Moreover, while the rate of postoperative complications (e.g arm and shoulder dysfunction, persistent pain, lymphedema or paresthesia, seroma or hematoma formation) decreased in $6-30 \%$ of patients treated with SLNB, the avoidance of ALND in women with negative SN status has progressively been adopted as the mainstay treatment for localized breast tumors. (3)

The SLN biopsy was initially presented by Gould in 1951 that performed a total parotidectomy for parotid cancer and discovered a positive LN localized at the intersection of the anterior and posterior facial veins. It has beenfurther used in the treatment of malignant melanoma and penile cancer. In the late 1990s, the SLN was described in breast cancer as the first axillary LN that drains the primary tumor, and it should be removed because it functions as a filter for the malignant cells avoiding dissemination into the regional LN. (4)

Two methods have been proposed for the removal of the SLN (5):

- intraoperatively, the surgeon injects a blue dye (patent blue, isosulfan blue, and methylene blue) into the breast parenchyma, performs an axillary incision at the level of the hair line and then looks for and removes the blue appearing node(s).

- with the use of a Gamma probe which helps detection and removal of the ALNs after the injection of a radioactive colloid material (technetium labelled sulfur colloid and albumin) into the breast.

Performing the combined method is recommended as it increases the rate of SLN detection and decreases the rate of false-negative results.

If the clinical, radiological and cytologic examinations show no axillary involvement then the axilla is clinically negative and SLN biopsy is primarily indicated. On the other hand, if suspicious node(s) are present in the axilla then SLB is contraindicated, an ultrasound (US) guided fine needle aspiration biopsy being further required in order to exclude ALN metastases. Even though the US guided fine needle aspiration biopsy is negative, during the performance of SLNB, all the macroscopic LN should be removed even though they do not take up blue dye or radiocolloid material.

Another contraindication for SLNB performance are previous surgical procedures to the breast and axilla as diagnostic methods such as: incisional or excisional biopsy, core needle biopsy under radiologic guidance, all of which increase the chance of a false-negative SLNB. The false negative rate of the SLNB ranges between 5\% and 10\% and less than $5 \%$ in experienced centers. (6)

For clinically negative axilla, if the SLNB is negative, an ALND can be omitted.

If the SLNB is positive, the probability of metastatic tumors in the axilla ranges between $40 \%$ and $50 \%$, hence requiring further ALND basing on the scientific evidence that axillary lymphadenectomy has a prognostic value and it has been advocated for determining staging and making treatment decisions. $(7,8)$ However, a significant number of patients could have axilla free disease although SLN status is positive. In this case, avoiding ALND could bring benefits in terms of postoperative complications (e.g lymphedema, cellulitis, wound and systemic infections), operative time and costs. (9) The AMAROS study (After mapping of the axilla: radiotherapy or surgery) demonstrated that the indication for adjuvant systemic chemotherapy is not influenced by the number of metastatic LN in the axilla. (10)

Taking into consideration the axillary tumor burden and the immune-histochemical characteristics of the tumor, a more individualized approach to the adjuvant treatment planning is recommended since the results of a recent clinical trial did not show any advantage in terms of either local control or survival in women with positive SLN status who underwent ALND. Although the therapeutic attitude has changed, ALND in patients with positive SLN should be still performed in selected cases. (11)

The role of this paper is to review the information on the role of ALND in the context of positive SLN biopsy, to assess the accuracy of SLNB after neoadjuvant chemotherapy and in locally recurrent breast cancer after surgical treatment with or without prior ALND.

\section{AXILLARY LYMPH NODE DISSECTION FOR MICRO- OR MACROMETASTATIC LN(S)}

The seventh edition of the American Joint Committee on Cancer (AJCC) manual, defined isolated tumor cells as cell clusters less than $0.2 \mathrm{~mm}$ in di- 
ameter or fewer than 200 tumor cells. On the other hand, micro-metastases are tumors with size between 0.2 and $2 \mathrm{~mm}$ or more than 200 tumor cells in number. Macro-metastasis includes nodes with more than $2 \mathrm{~mm}$ in size. The detection of isolated tumor cells in an ALN is being staged as NO while the presence of micro-and macrometastases is accepted as N1. (12)

Randomized controlled clinical trials have been performed in order to assess the impact of ALND on OS and DFS in patients with micro-metastases in the SLN(s). The IBCSG 23-01 study did not reveal any significant difference in terms of OS and DFS between two groups of women with micrometastases in the SLN who have been randomized to either axillary dissection or systemic therapy. DFS was $84 \%$ and $88 \%$ and OS $98 \%$ in both arms. (13) In the same way, data from NASP B-32 study with 10 years of follow-up revealed that the presence of occult metastases disease in the SLN(s) does influence neither the survival rate nor the rate of loco-regional recurrences. However, patients with micro-metastatic disease have a lower DFS survival compared with SLN negative patients and they can benefit from adjuvant therapy. (14)

On the other hand, the Netherland Cancer Registry reported a high percent of regional recurrences when ALND was not performed in patients with micro-metastases in axillary SLN, a shorter doubling time, grade 3 tumor, and negative hormone receptor status. Even in these patients, about 20\% can be expected to have additional axillary involvement. (15) As a result, ALND is avoided when isolated tumor cells or micro-metastases are detected in the SLN.

The need for completion of ALND in the case of patients with macro-metastases in the ALN(s) has been investigated in a randomized controlled trialACOSOG Z0011 in which 600 patients treated with breast conserving surgery, T1/T2 tumors and 1 or 2 positive macro-metastatic SLN(s) have been randomized into two groups: one who completed ALD following SLNB and one with SLNB alone. The median follow-up was 6.3 years and no significant difference in terms of OS and DFS has been noted between the two groups. (16) In a metaanalysis of 16 studies in which 3,268 patients with macro-metastases in the SLN(s) without completion of ALN were included, the rate of axillary recurrence was $0.7 \%$ after a median follow-up of 43 months. (17)

However, the low recurrence rates detected in the axilla when ALN is not further performed even though the SLN(s) are positive can be due to a suc- cessful adjuvant chemotherapy and radiotherapy which affect the axillary metastases and influence the decision on the therapeutic regimens. (6)

\section{AVOIDING OR PERFORMING ALND AFTER A POSITIVE SLNB?}

Basing on the information from the American College of Surgeons Oncology Group (ACOSOG)Z0011 trial, the American Society of Clinical Oncology (ASCO) published a guideline on SLN biopsy for patients with breast cancer which contains indications for performing or omitting ALND after a positive SLNB (16).

These recommendations are summarized in Table 1 (18).

\section{TABLE 1}

\begin{tabular}{|l|l|l|}
\hline $\begin{array}{l}\text { ALND may be not } \\
\text { performed }\end{array}$ & $\begin{array}{l}\text { ALND should be the } \\
\text { standard but omission } \\
\text { could be considered in } \\
\text { selected cases }\end{array}$ & $\begin{array}{l}\text { Absolute } \\
\text { indications for } \\
\text { ALND }\end{array}$ \\
\hline T1 or T2 primary & Patient <65 years old & $\begin{array}{l}\text { T3, T4 or } \\
\text { inflammatory } \\
\text { primary }\end{array}$ \\
\hline $\begin{array}{l}1 \text { or } 2 \text { positive SLN } \\
\text { without extra- } \\
\text { capsular extension }\end{array}$ & $\begin{array}{l}\text { Biology other than } \\
\text { Luminal A }\end{array}$ & $\begin{array}{l}\text { > SLN and/or } \\
\text { extra-capsular } \\
\text { extension }\end{array}$ \\
\hline $\begin{array}{l}\text { Lumpectomy } \\
\text { and conventional } \\
\text { radiation therapy } \\
\text { planned }\end{array}$ & Lobular histology & $\begin{array}{l}\text { Mastectomy or } \\
\text { unconventional } \\
\text { radiation } \\
\text { therapy } \\
\text { planned }\end{array}$ \\
\hline $\begin{array}{l}\text { ER and PR positive, } \\
\text { Her2/Neu negative } \\
\text { (equivalent to } \\
\text { Luminal A) biology }\end{array}$ & & \\
\hline Patient >65 years & & \\
\hline Ductal histology & & \\
\hline
\end{tabular}

ALND should not be performed for patients who have T1 or T2 tumors, one or two positive axillary SLN without extra-capsular infiltration and are planned to receive breast conserving surgery followed by radiotherapy. The ASCO 2014 indications on ALND after positive SLNB are opposed to the prior recommendations to dissect the axillae of all SLN positive patients. On the other hand, T3 or T4 tumors, inflammatory lesions with more than two positive SLN, mastectomy or unconventional radiation treatments are absolute contraindications for an ALD. $(16,18)$

The factors which can predict the risk of metastases in the axillary $\mathrm{LN}(\mathrm{s})$ are (19):

- a tumor size $>2 \mathrm{~cm}$;

- presence of macro-metastases with extracapsular extension in the SLN; 
- more than two SLN and more than 50\% positive $\mathrm{SLN}(\mathrm{s})$;

- presence of lympho-vascular space invasion.

Similarly, other studies showed that patients with T2 disease and two positive SLN(s) have higher probabilities of metastases in the ALN(s). A patient with a grade III tumor of $5 \mathrm{~cm}$ in size, and two positive SLN(s) may have a probability of over $90 \%$ for additional positive axillary LN(s). (20) However, the risk of additional metastases in the $\mathrm{ALN}(\mathrm{s})$ is not yet the only determinant of risk of recurrence. For example in study of 47 patients with positive SLN(s) and a risk of $11.5 \%$ of additional LN positivity only two patients $(4.2 \%)$ presented an axillary recurrence (21). However, due to their low sensitivity, specificity, and predictive value, these results cannot be applied in the clinical practice.

The results of the Z0011 trial are extracted from patients who underwent partial mastectomy, so the results are not available if the patient has an indication for total mastectomy. Furthermore, there are other subtypes of cancer which do not fulfill the criteria of the Z0011 trial. There is little scientific evidence in order to agree that ALND should not be performed in triple negative and Her2/Neu positive breast cancers. The triple negative breast cancer has a high regional and distant metastatic potential and there are no targeted therapies to control residual disease. Therefore, it must be treated from the most appropriate surgical point of view. In the case of Her $2 / \mathrm{Neu}$ positive breast cancers, it has not been proved that performing an ALND could control residual disease burden in the axilla. (22)

In the same way, the lobular breast carcinoma is more often multifocal and tends to metastasize to the gastrointestinal tract and the peritoneal surface. In this case, ALND could be omitted in particularly cases. For example, the study of the Surveillance, Epidemiology and End Results database demonstrated that there are no differences in terms of OS and DFS between patients with lobular carcinoma, $\mathrm{T} 1$ and ER positive tumors, less than $2 \mathrm{~cm}$ in size, and one positive SLN who did or did not undergo completion ALND. (23)

The available data from the Z0011 trial has however some limitations. The follow-up period of 6 years is too short as the subtype of tumors included in the trial-ER positive and Her2/Neu negative tumors- have a slow progression and a long metastases-free interval. Even though no significant differences in terms of OS, DFS and local recurrences have been observed between the two groups, definite conclusions with regard to per- forming or omitting ALND in positive SLN(s) patients cannot still be reached. $(6,18)$

\section{ROLE OF SLNB IN THE SETTING OF NEO-ADJUVANT CHEMOTHERAPY}

Neo-adjuvant chemotherapy is usually offered before surgery for patients with stage II or III breast cancer and large primary tumors or positive axillary LN(s). Its role is to reduce the volume of the tumor in order to decrease the extent of the surgical treatment. As chemotherapy usually causes fibrosis of the lymphatic ducts, applying the SLN concept after completion of the neo-adjuvant chemotherapy may result in higher rates of false-negative results. (24) The SENTINA (SENTinel NeoAdjuvant) multicenter study investigated the value of SLNB before and after neoadjuvant chemotherapy and concluded that SLNB should be used with caution after neo-adjuvant chemotherapy, as the false-negative rate of SLNB performed after systemic chemotherapy was $24.3 \%$ for women with one SLN removed, and $18.5 \%$ for those who had two SLN removed, both rates being higher than the pre-determined threshold of $10 \%$. (25)

Recently, it has been suggested that the rate of false-negative results could be reduced by sampling more than 3 SLN(s) using a dual-agent mapping technique with blue dye and radioactive colloid in order to improve the success of the procedure. However, the removal of three SLN after neo-adjuvant chemotherapy for each patient is not every time possible.

The current data has proven that it is hard to identify SLN(s) after neo-adjuvant chemotherapy. Furthermore, there is no information on local recurrence after SLNB following neo-adjuvant chemotherapy. Performing SLNB for patients who are node-negative before the initiation of chemotherapy is justified, whereas the available scientific evidence with regard to SLNB in node-positive patients who are converted to node-negative after neo-adjuvant chemotherapy is still not convincing. (24)

\section{SLNB IN THE CONTEXT OF LOCALLY RECURRENT BREAST CANCER}

Axillary dissection is the method of choice to obtain loco-regional control and determining prognosis in breast cancer patients. In locally recurrent breast cancer, the lymphatic channels may be remodeled following surgery, ALND or radiotherapy, so that the lymphatic drainage pathways may be no 
longer to the ipsilateral axilla, but to the intra-mammary $\mathrm{LN}(\mathrm{s})$, the contralateral axilla, or other regional locations. In this case, conventional imaging techniques and SLNB to the ipsilateral axilla may not identify metastases outside the ipsilateral axilla.

Lymphatic mapping with SLNB performed by dual technique is feasible and accurate for restaging the axilla in recurrent breast cancer patients. In this way, the identification rate of the SLN(s) improves and extra-axillary drainage pathways can be better identified. Moreover, the results obtained from SLNB can change the adjuvant treatment plan. $(24,26)$

\section{CONCLUSIONS}

The pathologic status of the axilla has a decisive effect on the adjuvant treatment plan. Over the past years, the introduction of the SLN concept has modified the surgical treatment of the axilla, the avoidance of the ALND resulting in a lower morbidity rate and a better quality of life in patients with early breast cancer.

Micro-metastases in the SLN(s) are no longer an indication for axillary dissection.

Although the major clinical trials have refined the indications for SLNB, ALND cannot be totally abandoned in all patients with $\mathrm{T} 1$ or $\mathrm{T} 2$ disease and up to two positive SLN, who are planned to receive lumpectomy and whole breast radiotherapy.

Only SLNB without ALND can be performed in women older than 65 years, with clinically negative axilla, ductal carcinomas on histology sections, no extra-capsular extension and ER/PR positive breast tumor. Axillary dissection is indicated in patients who undergo mastectomy or who do not receive postoperative radiotherapy of the affected breast. In the rest of the cases, especially when other subtypes of breast cancer are confirmed (e.g triple negative, Her 2/Neu positive, lobular carcinoma), a more individualized approach with involvement of the patient, who needs to be informed of the potential risks and benefits, is recommended.

With regard to the role of SLNB in the context of neo-adjuvant chemotherapy, for patients with positive LN status before neo-adjuvant chemotherapy, SLNB presents a high false-negative rate so that ALND is required after systemic therapy. If the axilla is clinically negative before neo-adjuvant chemotherapy, then SLNB is recommended.

However, more clinical trials and aggregate evidence are required to establish the exact group of women for whom the omission of ALND is safe and the oncological outcomes in view of risk-benefit ratio are favorable.

\section{REFERENCES}

1. Fisher B. The evolution of paradigms for the management of breast cancer: a personal perspective. Cancer Res 1992; 52(9):2371-2383

2. Patey D.H., Dyson W.H. The prognosis of carcinoma of the breast in relation to the type of operation performed. Br J Cancer 1948; 2(1):7-13

3. Krag D.N., Anderson S.J., Julian T.B., Brown A.M., Harlow S.P., Costantino J.P., Ashikaga T., Weaver D.L., Mamounas E.P., Jalovec L.M., Frazier T.G., Noyes R.D., Robidoux A., Scarth H.M., Wolmark N. Sentinel-lymph-node resection compared with conventional axillary-lymph-node dissection in clinically node-negative patients with breast cancer: overall survival findings from the NSABP B-32 randomised phase 3 trial. Lancet Oncol 2010; 11(10):927-933.

4. Gould E.A., Winship T., Philbin P.H., Kerr H.H. Observations on a "sentinel node" in cancer of the parotid. Cancer 1960; 13:77-78

5. Tanis P.J., Nieweg O.E., Valdes Olmos R.A., Th Rutgers E.J., Kroon B.B. History of sentinel node and validation of the technique. Breast Cancer Res 2001; 3(2):109-112

6. Atalay $\mathbf{C}$. New concepts in axillary management of breast cancer. World J Clin Oncol 2014; 5(5):895-900

7. Ram R., Singh J., McCaig E. Sentinel Node Biopsy Alone versus Completion Axillary Node Dissection in Node Positive Breast Cancer: Systematic Review and Meta-Analysis. Int J Breast Cancer 2014; 2014:513780

8. Kuerer H.M., Newman L.A. Lymphatic mapping and sentinel lymph node biopsy for breast cancer: developments and resolving controversies. J Clin Oncol 2005; 23(8):1698-1705
9. DiSipio T., Rye S., Newman B., Hayes S. Incidence of unilateral arm lymphoedema after breast cancer: a systematic review and meta-analysis. Lancet Oncol 2013; 14(6):500-515

10. Straver M.E., Meijnen P., van Tienhoven G., van de Velde C.J., Mansel R.E., Bogaerts J., Demonty G., Duez N., Cataliotti L., Klinkenbijl J., Westenberg H.A., van der M.H., Hurkmans C., Rutgers E.J. Role of axillary clearance after a tumor-positive sentinel node in the administration of adjuvant therapy in early breast cancer. J Clin Oncol 2010; 28(5):731-737

11. Acea B., Calvo M.L., Antolin N.S., Albaina L.L., Juaneda M.M., Santiago F.P., Silva R.C., Mosquera O.J., Varela R., Jr., Soler F.R., Facio V.I., Candal C.I., Cereijo G.C. (Proposal for a new multidisciplinary therapeutic strategy in the breast cancer patient with sentinel lymph node metastasis). Cir Esp 2012; 90(10):626-633.

12. Edge S.B. American Joint Committee on Cancer: AJCC Cancer Staging Manual. 7th ed. New York: Springer, 2009

13. Galimberti V., Cole B.F., Zurrida S., Viale G., Luini A., Veronesi P., Baratella P., Chifu C., Sargenti M., Intra M., Gentilini O., Mastropasqua M.G., Mazzarol G., Massarut S., Garbay J.R., Zgajnar J., Galatius H., Recalcati A., Littlejohn D., Bamert M., Colleoni M., Price K.N., Regan M.M., Goldhirsch A.,et al. Axillary dissection versus no axillary dissection in patients with sentinel-node micrometastases (IBCSG 23-01): a phase 3 randomised controlled trial. Lancet Oncol 2013; 14(4):297-305

14. Julian T.B., Anderson S.J., Krag D.N., et al. "10-yr follow-up results of occult detected sentinel node disease: NSABP B-32, a randomized 
phase III clinical trial to compare sentinel node resection (SNR) to conventional axillary dissection $(A D)$ in clinically node-negative breast cancer patients," in Proceedings of the San Antonio Breast Cancer Symposium, Abstract S2-05, San Antonio, Tex, USA, 2013

15. Pepels M.J., de Boer M., Bult P., van Dijck J.A., van Deurzen C.H., Menke-Pluymers M.B., van Diest P.J., Borm G.F., Tjan-Heijnen V.C. Regional recurrence in breast cancer patients with sentinel node micrometastases and isolated tumor cells. Ann Surg 2012; 255(1):116-121

16. Giuliano A.E., McCall L., Beitsch P., Whitworth P.W., Blumencranz P., Leitch A.M., Saha S., Hunt K.K., Morrow M., Ballman K. Locoregional recurrence after sentinel lymph node dissection with or without axillary dissection in patients with sentinel lymph node metastases: the American College of Surgeons Oncology Group Z0011 randomized trial. Ann Surg 2010; 252(3):426-432

17. Francissen C.M., Dings P.J., van Dalen T., Strobbe L.J., van Laarhoven H.W., de Wilt J.H. Axillary recurrence after a tumorpositive sentinel lymph node biopsy without axillary treatment: a review of the literature. Ann Surg Oncol 2012; 19(13):4140-4149

18. Voutsadakis I.A., Spadafora S. Axillary lymph node management in breast cancer with positive sentinel lymph node biopsy. World J Clin Oncol 2015; 6(1):1-6

19. van la Parra R.F., Peer P.G., Ernst M.F., Bosscha K. Meta-analysis of predictive factors for non-sentinel lymph node metastases in breast cancer patients with a positive SLN. Eur J Surg Oncol 2011; 37(4):290-299

20. Van Zee K.J., Manasseh D.M., Bevilacqua J.L., Boolbol S.K., Fey J.V., Tan L.K., Borgen P.I., Cody H.S., III, Kattan M.W. A nomogram for predicting the likelihood of additional nodal metastases in breast cancer patients with a positive sentinel node biopsy. Ann Surg Oncol 2003; 10(10):1140-1151

21. Yegiyants S., Romero L.M., Haigh P.I., DiFronzo L.A. Completion axillary lymph node dissection not required for regional control in patients with breast cancer who have micrometastases in a sentinel node. Arch Surg 2010; 145(6):564-569

22. Slamon D.J., Leyland-Jones B., Shak S., Fuchs H., Paton V., Bajamonde A., Fleming T., Eiermann W., Wolter J., Pegram M., Baselga J., Norton L. Use of chemotherapy plus a monoclonal antibody against HER2 for metastatic breast cancer that overexpresses HER2. N Engl J Med 2001; 344(11):783-792

23. Wang J., Mittendorf E.A., Sahin A.A., Yi M., Caudle A., Hunt K.K., Wu Y. Outcomes of sentinel lymph node dissection alone vs. axillary lymph node dissection in early stage invasive lobular carcinoma: a retrospective study of the surveillance, epidemiology and end results (SEER) database. PLoS One 2014; 9(2):e89778

24. Assi H., Sbaity E., Abdelsalam M., Shamseddine A. Controversial indications for sentinel lymph node biopsy in breast cancer patients. Biomed Res Int 2015; 2015:405949

25. Kuehn T., Bauerfeind I., Fehm T., Fleige B., Hausschild M., Helms G., Lebeau A., Liedtke C., von Minckwitz G., Nekljudova V., Schmatloch S., Schrenk P., Staebler A., Untch M. Sentinel-lymphnode biopsy in patients with breast cancer before and after neoadjuvant chemotherapy (SENTINA): a prospective, multicentre cohort study. Lancet Oncol 2013; 14(7):609-618

26. Taback B., Nguyen P., Hansen N., Edwards G.K., Conway K., Giuliano A.E. Sentinel lymph node biopsy for local recurrence of breast cancer after breast-conserving therapy. Ann Surg Oncol 2006; 13(8):1099-1104 